TRANSACTIONS OF THE

AMERICAN MATHEMATICAL SOCIETY

Volume 349, Number 2, February 1997, Pages 641-655

S 0002-9947(97)01591-2

\title{
LOCALLY CONFORMAL KÄHLER STRUCTURES IN QUATERNIONIC GEOMETRY
}

\author{
LIVIU ORNEA AND PAOLO PICCINNI
}

\begin{abstract}
We consider compact locally conformal quaternion Kähler manifolds $M$. This structure defines on $M$ a canonical foliation, which we assume to have compact leaves. We prove that the local quaternion Kähler metrics are Ricci-flat and allow us to project $M$ over a quaternion Kähler orbifold $N$ with fibers conformally flat 4-dimensional real Hopf manifolds. This fibration was known for the subclass of locally conformal hyperkähler manifolds; in this case we make some observations on the fibers' structure and obtain restrictions on the Betti numbers. In the homogeneous case $N$ is shown to be a manifold and this allows a classification. Examples of locally conformal quaternion Kähler manifolds (some with a global complex structure, some locally conformal hyperkähler) are the Hopf manifolds quotients of $\mathbb{H}^{n}-\{0\}$ by the diagonal action of appropriately chosen discrete subgroups of $\mathrm{CO}^{+}(4)$.
\end{abstract}

\section{INTRODUCTION}

The oldest and simplest example of a complex manifold which does not admit any Kähler metric is the Hopf manifold $H_{\mathbb{C}}=\left(\mathbb{C}^{n}-\{0\}\right) / \Gamma_{2}, \Gamma_{2}$ generated by $\left(z_{1}, \ldots, z_{n}\right) \mapsto\left(2 z_{1}, \ldots, 2 z_{n}\right)$. Since the conformal Kähler metric $g=\left(\sum_{\alpha} z_{\alpha} \bar{z}_{\alpha}\right)^{-1}$ $\cdot \sum_{\alpha} d z_{\alpha} \otimes d \bar{z}_{\alpha}$ on $\mathbb{C}^{n}-\{0\}$ is invariant by $\Gamma_{2}, g$ induces a locally conformal Kähler metric on $H_{\mathbb{C}}$. The theory of locally conformal Kähler manifolds, initiated by I. Vaisman in 1976 and by now motivated by several significant examples, has been developed with more conclusive results for the subclass of generalized Hopf manifolds [Va1, Va3]. These are locally conformal Kähler manifolds with the parallelism condition of their Lee form, 1-form canonically associated to the structure. A survey of this theory is given in [Or].

In quaternionic geometry one can consider the Hopf manifold $H_{\mathbb{H}}=$ $\left(\mathbb{H}^{n}-\{0\}\right) / \Gamma_{2}$ as one of the simplest examples of a hypercomplex manifold not admitting any hyperkähler metric. The same $g$ as before (in quaternionic coordinates) is a locally conformal hyperkähler metric. In fact, the two well-known quaternionic analogues of complex Kähler manifolds, namely hyperkähler manifolds and quaternion Kähler manifolds, have as locally conformal correspondents the two classes which are the subject of this paper: locally conformal hyperkähler manifolds and the larger class of locally conformal quaternion Kähler manifolds.

Received by the editors September 1, 1994.

1991 Mathematics Subject Classification. Primary 53C15, 53C25, 53C55.

Key words and phrases. Locally conformal hyperkähler manifold, locally conformal quaternion Kähler manifold, Einstein-Weyl structure.

The first author was supported by C.N.R. of Italy, the second author by M.U.R.S.T. of Italy and by the E. Schrödinger Institute in Vienna. 
Examples of both can actually be obtained by admitting more general groups than $\Gamma_{2}$ to act on $\mathbb{H}^{n}-\{0\}$ (cf. $\S 6$ ).

These two classes of quaternionic manifolds have already appeared in the contexts of Hermitian-Einstein-Weyl structures [Pe-Po-Sw] and of 3-Sasakian structures [Bo-Ga-Ma1]. A special feature of this quaternionic setting, of particular interest for us, is the possibility of assuming the Lee form to be parallel. This possibility, not guaranteed in the complex case, depends here on the Einstein property of the local conformal metrics applied to a recent result of P. Gauduchon [Gau2].

Accordingly, the structure of compact locally conformal hyperkähler and locally conformal quaternion Kähler manifolds is described by fibrations over orbifolds carrying different structures, similarly to the case of complex generalized Hopf manifolds (cf. Theorems 2.1, 3.8, 5.1, that refine earlier results in [Pe-Po-Sw]). However, the interplay with other geometries - quaternion Kähler, complex Kähler via twistors, 3-Sasakian, local similarity-appears here to be richer than in the complex case. The most significant fibration, with base a quaternion Kähler orbifold, has as fibers 4-dimensional compact manifolds of Hopf type, with universal covering $\mathbb{R}^{4}-\{0\}$ and endowed, according to the cases, with a complex, hypercomplex, or quaternionic structure (cf. Proposition 6.1). This relates with the work of Ma. Kato [Ka1], [Ka2] on discrete groups acting on $\mathbb{C}^{2}-\{0\}$ to preserve its complex or hypercomplex structure.

The locally conformal hyperkähler manifolds carry naturally a $S^{2}$-bundle of compatible complex locally conformal Kähler structures. Yet, their Betti numbers have here much stronger restrictions than in the complex case (cf. Theorems 3.11 and Remarks $3.12,3.13)$ : for example it is necessary that $b_{1}=1$, whether examples exist of a complex generalized Hopf manifold for any odd $b_{1}$. Also, none of the compatible complex structure may support a Kähler metric. Finally, for compact locally conformal hyperkähler homogeneous manifolds, the quaternion Kähler orbifold base of the fibration in Hopf surfaces is actually a homogeneous manifold: this, similarly to the case of 3-Sasakian manifolds [Bo-Ga-Ma1], allows a classification (cf. Proposition 4.2 and Corollary 4.3).

\section{Preliminaries}

We recall the following definition from complex geometry. A complex Hermitian manifold $\left(M^{2 n}, g, J\right)$ is locally conformal Kähler (l.c.K.) if over open neighbourhoods $\left\{U_{i}\right\}$ covering $M,\left.g\right|_{U_{i}}=e^{f_{i}} g_{i}^{\prime}$ with $g_{i}^{\prime}$ Kähler and $f_{i}$ a $\mathcal{C}^{\infty}$ function on $U_{i}$. This is equivalent to the condition:

$$
d \Omega=\omega \wedge \Omega, \quad d \omega=0,
$$

where $\Omega$ is the Kähler form of $g$ and the closed 1-form $\omega$, locally defined by $\left.\omega\right|_{U_{i}}=$ $d f_{i}$, is called the Lee form. Hence, $\omega$ is exact iff $M$ is globally conformal Kähler (g.c.K.). The Levi-Civita connection $D^{i}$ of the local Kähler metrics $g_{i}^{\prime}$ glue together on $M$ to a connection $D$ related to the Levi-Civita connection $\nabla$ of $g$ by the formula

$$
D_{X} Y=\nabla_{X} Y-\frac{1}{2}\{\omega(X) Y+\omega(Y) X-g(X, Y) B\},
$$

where $B=\omega^{\natural}$ is the Lee vector field. The two properties $D g=\omega \otimes g$ and $D$ torsion free show that $D$ is a Weyl connection of the conformal manifold $(M,[g])$ satisfying, in addition, $D J=0$. Moreover, the associated 1-form of the Weyl manifold $(M,[g], D)$ is precisely the Lee form $\omega$. 
A significant subclass of l.c.K. manifolds is given by the generalized Hopf manifolds (see [Va4]) defined by the stronger condition $\nabla \omega=0$. Note that on compact $M$ this is not compatible with $\omega=d f$, i.e. with the g.c.K. condition. Moreover, on compact $M$, the generalized Hopf condition implies the vanishing of the Euler characteristic; thus not all manifolds carrying a l.c.K. metric may admit a generalized Hopf one. The following result yields a sufficient condition for a l.c.K. metric to be globally conformal with a generalized Hopf one:

Theorem 1.3 ([Gau1], [Gau2]). Let $\left(M^{n},[g], D\right)$ be a compact Weyl manifold, $n \geq$ 3.

(i) There exists a metric $g_{0} \in[g]$, unique up to homotheties, whose associated 1-form $\omega_{0}$ is $g_{0}$-coclosed.

(ii) If, in addition, $\left(M^{n},[g], D\right)$ is Einstein-Weyl (i.e. the symmetrized Ricci tensor of $D$ is proportional to $g$ ) and $\omega_{0}$ is closed but not exact, then $\nabla^{0} \omega_{0}=0$ with respect to the Levi-Civita connection $\nabla^{0}$ of $g^{0}$.

To approach our subject, we recall the following terminology. Let $M$ be a $4 n$ dimensional $\mathcal{C}^{\infty}$ manifold. A triple $I_{1}, I_{2}, I_{3}$ of global integrable complex structures on $M$ satisfying the quaternionic identities: $I_{\alpha} I_{\beta}=I_{\gamma}$ for $(\alpha, \beta, \gamma)=(1,2,3)$ and cyclic permutations, defines a hypercomplex structure on $M$. If a Riemannian metric $g$ is added, assumed to be Hermitian with respect to $I_{1}, I_{2}, I_{3}$, one gets a hyperhermitian manifold $\left(M, g, I_{1}, I_{2}, I_{3}\right)$.

More generally, by $(M, g, H)$ we denote a quaternion Hermitian manifold. Here $H$ is a rank 3 subbundle of $\operatorname{End}(T M)$, locally spanned by (not necessarily integrable) almost complex structures $I_{1}, I_{2}, I_{3}$, again satisfying the quaternionic identities and related on the intersections of trivializing open sets by matrices of $S O(3)$. $H$ defines on $M$ a structure of quaternionic manifold and the local almost complex structures $I_{1}, I_{2}, I_{3}$ are said to be compatible with the quaternionic structure $H$. The additional datum of a metric $g$, Hermitian with respect to the local compatible almost complex structures, defines the quaternion Hermitian manifold $(M, g, H)$. Recall that the hyperhermitian or quaternion Hermitian metric $g$ is said to be $h y$ perkähler or quaternion Kähler if its Levi-Civita connection $\nabla$ satisfies respectively $\nabla I_{\alpha}=0(\alpha=1,2,3)$ or $\nabla H \subset H$. We can now define the two classes of manifolds that we will be concerned with in this paper.

Definition 1.4. (i) A hyperhermitian manifold ( $\left.M, g, I_{1}, I_{2}, I_{3}\right)$ is locally conformal hyperkähler (l.c.h.K.) if, over open neighbourhoods $\left\{U_{i}\right\}$ covering $M,\left.g\right|_{U_{i}}=$ $e^{f_{i}} g_{i}^{\prime}$ with $g_{i}^{\prime}$ quaternion Kähler on $U_{i}$.

(ii) A quaternion Hermitian manifold $(M, g, H)$ is locally conformal quaternion Kähler (l.c.q.K.) if, over open neighbourhoods $\left\{U_{i}\right\}$ covering $M,\left.g\right|_{U_{i}}=e^{f_{i}} g_{i}^{\prime}$ with $g_{i}^{\prime}$ quaternion Kähler on $u_{i}$.

In both cases we have a Lee form $\omega$, locally defined by $\left.\omega\right|_{U_{i}}=d f_{i}$ and satisfying:

$$
d \Theta=\omega \wedge \Theta, \quad d \omega=0,
$$

where $\Theta=\sum_{\alpha=1,2,3} \Omega_{\alpha} \wedge \Omega_{\alpha}$ is the (global) Kähler 4-form. Properties (1.5) for $\Theta$ are also sufficient for a hyperhermitian or quaternion Hermitian metric to be l.c.h.K. or l.c.q.K., respectively.

Then, recalling that hyperkähler and quaternion Kähler metrics are Einstein, Gauduchon's Theorem 1.3 can be applied to our context. Precisely: 
Corollary 1.6. Let $(M, g)$ be a compact locally conformal hyperkähler or locally conformal quaternion Kähler manifold and assume that no metric in the conformal class $[g]$ of $g$ is respectively hyperkähler or quaternion Kähler. Then there exists a $g_{0} \in[g]$ whose Lee form $\omega_{0}$ is $\nabla^{0}$-parallel.

We point out that the possibility of assuming $\nabla \omega=0$, given by Corollary 1.6, has no correspondent in the complex case of l.c.K. manifolds; the standard Hopf surface $H_{\mathbb{C}}$ blown up at one point has nonzero Euler characteristic and is therefore an example of a l.c.K. manifold not admitting any generalized Hopf metric.

Throughout the paper (with the only exception of Proposition 2.3) by the terms locally conformal hyperkähler or locally conformal quaternion Kähler structures we mean such structures that are not globally conformal ones. Moreover, by the above discussion, the following assumptions are not restrictive for compact manifolds and will always by made:

(i) the fixed metric $g$ makes $\omega$ parallel: $\nabla \omega=0$;

(ii) $\|\omega\|=1$.

Proposition 1.7. Let $\left(M, g, I_{1}, I_{2}, I_{3}\right)$ be a locally conformal hyperkähler manifold which is either compact or satisfying $\nabla \omega=0$; let $B=\omega^{\natural}$ be its Lee vector field and $\Omega_{\alpha}$ the Kähler 2-form with respect to $I_{\alpha}$. Then the following formulas hold good:

$$
\begin{gathered}
\nabla B=0, \quad L_{B} I_{\alpha}=0, \quad L_{B} g=0, \quad L_{B} \Omega_{\alpha}=0, \quad L_{B} \Theta=0, \\
\left(\nabla_{X} I_{\alpha}\right) Y=\frac{1}{2}\left\{\omega\left(I_{\alpha} Y\right) X-\omega(Y) I_{\alpha} X-g\left(X, I_{\alpha} Y\right) B+g(X, Y) I_{\alpha} B\right\}, \\
L_{I_{\alpha} B} I_{\alpha}=0, \quad L_{I_{\alpha} B} I_{\beta}=I_{\gamma}, \quad L_{I_{\alpha} B} g=0, \\
{\left[B, I_{\alpha} B\right]=0, \quad\left[I_{\alpha} B, I_{\beta} B\right]=I_{\gamma} B,} \\
\left(\nabla_{X} I_{\alpha} \omega\right) Y=\frac{1}{2}\left\{I_{\alpha} \omega(Y) \omega(X)-\omega(Y) I_{\alpha} \omega(X)-g\left(X, I_{\alpha} Y\right)\right\}, \\
d I_{\alpha} \omega=2 \nabla_{X} I_{\alpha} \omega=\Omega_{\alpha}-\omega \wedge I_{\alpha} \omega, \\
L_{I_{\alpha} B} \Omega_{\alpha}=0, \quad L_{I_{\alpha} B} \Omega_{\beta}=\Omega_{\gamma}, \quad L_{I_{\alpha} B} \Theta=0,
\end{gathered}
$$

where $L_{X}$ is the Lie derivative and $(\alpha, \beta, \gamma)=(1,2,3)$ and cyclic permutations.

Proof. All the formulas are easily obtained from the case of complex generalized Hopf manifolds (cf. [Ch-Pi], [Or], [Va3]) by choosing on the l.c.h.K. manifold the appropriate compatible complex structure. In particular, from (1.7.a), (1.7.c) we see that $B, I_{\alpha} B$ are infinitesimal automorphisms of the quaternion Hermitian structure $H \subset \operatorname{End}(T M)$ of $M$, here globally spanned by $I_{1}, I_{2}, I_{3}$ (cf. [Pi]).

For a l.c.q.K. manifold, the Weyl connection $D$ can still be defined by the formula (1.2) in terms of the Lee 1-form of the structure. Yet $D$ does not preserve in this case the compatible almost complex structures individually but only their 3-dimensional bundle $H$. In fact [Pe-Po-Sw]:

$$
D I_{\lambda}=\sum a_{\lambda_{\mu}} \otimes I_{\mu}
$$

where $\lambda, \mu=1,2,3$ and $\left(a_{\lambda \mu}\right)$ is a skew-symmetric matrix of local 1-forms. Accordingly, formulas (1.7.a)-(1.7.g) have corresponding l.c.q.K. ones (1.7.a')-(1.7.g'). For example, $\left(1.7 . \mathrm{b}^{\prime}\right)$ is obtained by adding $\left(D_{X} I_{\alpha}\right) Y=\sum_{\mu} a_{\alpha \mu}(X) I_{\mu} Y$ in the righthand side of (1.7.b).

We shall be interested in the properties of some canonically defined foliations $\mathcal{F}$ on l.c.h.K. and l.c.q.K. manifolds. Some convenient hypotheses on $\mathcal{F}$ have to 
be chosen in order to relate the geometry of $M$ to that of the leaf space $M / \mathcal{F}$. Two natural assumptions on $\mathcal{F}$ are: (i) $\mathcal{F}$ is regular in the sense of R. Palais (cf. $[\mathrm{Pa}]$ ), assuring that $M / \mathcal{F}$ is a $\mathcal{C}^{\infty}$ manifold; (ii) $\mathcal{F}$ has compact leaves, implying that $M / \mathcal{F}$ is an orbifold, i.e. a second countable Hausdorff space locally modelled on finite quotients of $\mathbb{R}^{n}$. We shall make on all foliations $\mathcal{F}$ the hypothesis (ii). This is, on one hand, a weaker assumption, providing more examples (see [Bo-Ga-Ma1]) and on the other hand orbifolds seem to be a natural class to work with in quaternionic geometry.

We recall that a point $p$ of an orbifold $N$ is said to be regular if it has a neighborhood homeomorphic with $\mathbb{R}^{n}$, and singular if such a neighborhood does not exist. We refer to $[\mathrm{Mo}]$ for the formal definition and properties of orbifolds and to [Sat] for the generalization of geometric objects to the orbifold category.

\section{LOCALLY CONFORMAL QUATERNION KÄHLER MANIFOLDS}

Let $M$ be a compact l.c.q.K. manifold. As mentioned, we choose the metric $g$ such that $\nabla \omega=0$ and $\|\omega\|=1$. The vector fields $B, I_{1} B, I_{2} B, I_{3} B$ span a 4dimensional distribution $\mathcal{D}$ that, by formula (1.7.d), is seen to be integrable (cf. $[\mathrm{Pe}-\mathrm{Po}-\mathrm{Sw}])$. Then:

Theorem 2.1. On a compact locally conformal quaternion Kähler $(M, H, g)$, all the local quaternion Kähler metrics are Ricci-flat and $M$ is therefore locally conformal locally hyperkähler. Moreover, the metric $g$ is bundle-like with respect to $\mathcal{D}$ and, if $\mathcal{D}$ has compact leaves, projects to an Einstein metric with positive scalar curvature at the regular points of the orbifold $N=M / \mathcal{D}$.

Proof. The vanishing of the scalar curvature $s^{D}$ follows from Theorem 3 in [Gau2]. A direct proof in this context can be given as follows. The scalar curvatures $s$ of $g$ and $s_{i}^{D}$ of the quaternion Kähler $g_{i}^{\prime}$ on $U_{i}$ are related by

$$
s_{i}^{D}=e^{-f_{i}}\left[\left.s\right|_{U_{i}}-\frac{(4 n-1)(2 n-1)}{2}\right]
$$

(cf. [Be], p. 59). It follows that $s_{i}^{D}=$ constant; thus, if $s_{i}^{D} \neq 0$, after derivation:

$$
\omega_{i}=d \ln \left[\left.s\right|_{U_{i}}-\frac{(4 n-1)(2 n-1)}{2}\right] .
$$

Thus, since both $\omega$ and $s$ are global on $M, \omega$ is exact, in contradiction with the assumption made after Corollary 1.6. If $s_{i}^{D}=0$ on some $U_{i}$, then $s=\left.s\right|_{U_{i}}=$ $\frac{(4 n-1)(2 n-1)}{2}$, constant on $M$; hence $s_{i}^{D}=0$ for all $U_{i}$. Then all local Kähler metrics are Ricci-flat.

To see that $g$ is bundle-like, as $L_{B} g=0$ by (1.7.a), we only have to compute $L_{I_{\alpha} B} g$ on horizontal vector fields $X, Y$. By (1.7.b) we derive

$$
\begin{aligned}
\left(L_{I_{\alpha} B} g\right)(X, Y) & =g\left(\nabla_{X} I_{\alpha} B, Y\right)+g\left(X, \nabla_{Y} I_{\alpha} B\right) \\
& =g\left(\left(\nabla_{X} I_{\alpha}\right) B, Y+g\left(X,\left(\nabla_{Y} I_{\alpha}\right) B\right)\right. \\
& =\sum_{\mu}\left\{a_{\alpha \mu}(X) I_{\mu} \omega(Y)+a_{\alpha \mu}(Y) I_{\mu} \omega(X)\right\}=0 .
\end{aligned}
$$


Therefore, at regular points, $N$ is a Riemannian manifold and the projection $M \rightarrow$ $N$ is a Riemannian submersion. The Ricci tensors of $M$ and $N$ are related by

$$
S^{N}(X, Y)=S^{M}(X, Y)+g\left(A_{X} B, A_{Y} B\right)+\sum_{\alpha=1}^{3} g\left(A_{X} I_{\alpha} B, A_{Y} I_{\alpha} B\right),
$$

where $A_{X}$ is the O'Neill tensor acting on vertical vector fields by $A_{X} V=h \nabla_{X} V, h$ being the horizontal projector (cf. [Be], p. 244). We have $A_{X} B=0$ and, from (1.7.b), $A_{X} I_{\alpha} B=-\frac{1}{2} I_{\alpha} X$. On the other hand, taking into account that the local quaternion Kähler metrics are Ricci-flat, on horizontal vector fields we have $S^{M}=\frac{2 n-1}{2} g$. Thus $S^{N}=\left(n+\frac{1}{4}\right) g^{N}$ and the proof is complete.

Remark 2.2. A consequence of the above result is that on compact l.c.q.K. manifolds one may always assume the neighbourhoods $U_{i}$ to be simply connected and the local compatible almost complex structures to be integrable and parallel. However, the existence of a global hypercomplex structure on $M$ is not implied (cf. examples in $\S 6)$. Note that with respect to local parallel compatible $I_{1}, I_{2}, I_{3}$ the matrix $\left(a_{\lambda \mu}\right)$ in formula (1.8) vanishes. Therefore, with this choice, formulas (1.7.a),..,(1.7.g) can be applied without modifications also to compact l.c.q.K. manifolds.

A characterization of quaternion Kähler manifolds in the larger class of l.c.q.K. manifolds (here not necessarily nonglobally conformal) is given as follows. Cf. [Va2] for the complex case.

Proposition 2.3. A locally conformal quaternion Kähler manifold $\left(M^{4 n}, g, H\right)$, $n \geq 2$, is quaternion Kähler if and only if through each point of it there exists a totally geodesic submanifold $Q$ of real dimension $4 h \geq 8$, which is quaternion Kähler with respect to the structure induced on it by $(g, H)$.

Proof. The relation between the second fundamental forms $b_{i}^{\prime}$ and $b$ of a submanifold with respect to the metrics $g_{i}^{\prime}$ and $g$ is easily seen to be

$$
b_{i}^{\prime}=b+g \otimes B^{\nu},
$$

where $B^{\nu}$ is the part of $B$ normal to the submanifold. Let $q \in M$ and $Q$ a quaternion Kähler submanifold as in the statement. Let $j$ be the immersion of $Q$ in $M$. Then $j^{*} d \Theta=0$; thus (cf. (1.5)) $j^{*} \omega \wedge j^{*} \Theta=0$. As $\operatorname{rank} j^{*} \Theta=4 h \geq 8$, necessarily $j^{*} \omega=0$. It follows that the Lee vector field $B$ is normal to $Q$; hence $B=B^{\nu}$. Further, $j^{*} \omega=0$ shows also that $Q \cap U_{i}$ is a quaternionic submanifold of the quaternion Kähler manifold $\left(U_{i}, g_{i}^{\prime}\right)$. Since quaternionic submanifolds of quaternion Kähler manifolds are known to be totally geodesic (cf. for example [Gr]), this is the case for $Q \cap U_{i}$ in $U_{i}$ with respect to $g_{i}^{\prime}$. Thus $b=-g \otimes B$ on $Q \cap U_{i}$. But $b=0$ from the assumption; thus $B=0$ on $Q \cap U_{i}$. Since such a $Q$ exists through any point $q \in M$, it follows that $B \equiv 0$ on $M$; i.e. $g$ is a quaternion Kähler metric. The converse part is clear with $Q=M$.

\section{LOCALLY CONFORMAL HYPERKÄHLER MANIFOLDS}

Let $M$ now be a compact l.c.h.K. manifold, $\mathcal{D}$ the 4-dimensional foliation on $M$ defined in $\S 2$ and $\mathcal{D}^{\perp}$ its orthogonal complement. The structure of $M$ has been described in [Pe-Po-Sw] and can be related to 3-Sasakian manifolds. The latter were extensively studied in the recent paper [Bo-Ga-Ma1]; their consideration goes back to the seventies, starting with [Ud] (who introduced them as coquaternionic manifolds) and $[\mathrm{Ku}]$. 
Proposition 3.1. (i) $\mathcal{D}$ is a Riemannian totally geodesic foliation. Its leaves, if compact, are complex Hopf surfaces admitting an integrable hypercomplex structure (cf. Theorem 3.2 below). In particular, they may be nonprimary.

(ii) $\mathcal{D}^{\perp}$ is not integrable. Its integral manifolds are totally real and have maximal real dimension $n-1$.

Proof. As in $\S 2, \mathcal{D}$ is integrable (1.7.d); here the vector fields $B, I_{\alpha} B$ are actually Killing (1.7.a), (1.7.c); thus $\mathcal{D}$ is Riemannian and by (1.7.b) totally geodesic. If the leaves are compact, their structure can be deduced by Theorem 1 in [Bo]. They are in fact tangent to the Lee vector field $B=\omega^{\natural}$, and hence carry a structure of hyperhermitian nonhyperkähler 4-dimensional manifold. As for (ii), observe that a submanifold $M^{\perp}$ of $M$ is an integral manifold of $\mathcal{D}^{\perp}$ if and only if $\omega$ and $I_{\alpha} \omega$ are zero on $M^{\perp}$. On such an integral submanifold $d I_{\alpha} \omega$ is then also zero. Then formula (1.7.f) implies that $I_{\alpha} X$ is normal to $M^{\perp}$ for all the $X$ tangent to $M^{\perp}$. The last assertion then follows.

Here integrable hypercomplex structure is intended in the sense of $G$-structures, i.e. of the existence of a local quaternionic coordinate such that the differential of the change of coordinate belongs to $\mathbb{H}^{*}$. For further use we recall the following:

Theorem 3.2 ([Ka2]). A complex Hopf surface $S$ admits an integrable hypercomplex structure if and only if $S=(\mathbb{H}-\{0\}) / \Gamma$ where the discrete group $\Gamma$ is conjugate in $G l(2, \mathbb{C})$ to any of the following subgroups $G \subset \mathbb{H}^{*} \subset G l(2, \mathbb{C})$ :

(i) $G=\mathbb{Z}_{m} \times \Gamma_{c}$ with $\mathbb{Z}_{m}$ and $\Gamma_{c}$ both cyclicly generated by left multiplication by $a_{m}=e^{2 \pi i / m}, m \geq 1$, and $c \in \mathbb{C}^{*}$.

(ii) $G=L \times \Gamma_{c}$, where $c \in \mathbb{R}^{*}$ and $L$ is one of the following: $D_{4 m}$, the dihedral group, $m \geq 2$, generated by the quaternion $j$ and $\rho_{m}=e^{\pi i / m} ; T_{24}$, the tetrahedral group generated by $\zeta^{2}$ and $\frac{1}{\sqrt{2}}\left(\zeta^{3}+\zeta^{3} j\right), \zeta=e^{\pi i / 4} ; O_{48}$, the octahedral group generated by $\zeta$ and $\frac{1}{\sqrt{2}}\left(\zeta^{3}+\zeta^{3} j\right) ; I_{120}$, the icosahedral group generated by

$$
\varepsilon^{3}, \quad j, \quad \frac{1}{\sqrt{5}}\left[\varepsilon^{4}-\varepsilon+\left(\varepsilon^{2}-\varepsilon^{3}\right) j\right], \quad \varepsilon=e^{2 \pi i / 5} .
$$

(iii) $G$ generated by $\mathbb{Z}_{m}$ and $c j, m \geq 3, c \in \mathbb{R}^{*}$.

(iv) $G$ generated by $D_{4 m}$ and $c \rho_{2 n}, c \in \mathbb{R}^{*}$ or by $T_{24}$ and $c \zeta, c \in \mathbb{R}^{*}$.

It follows from Theorem 2.1 that the leaf space $N=M / \mathcal{D}$ of a compact l.c.h.K. $M$ is, at regular points, an Einstein manifold with positive scalar curvature. Moreover, by (1.7.c) and (1.7.g) we see that, although no single element of $H$ projects under $p: M \rightarrow N$, this is the case for the bundle $H$ itself and the Kähler 4-form $\Theta$. Thus (cf. [Pe-Po-Sw]):

Proposition 3.3. Let $M$ be a compact locally conformal hyperkähler manifold of real $\operatorname{dim} \geq 12$ and such that $\mathcal{D}$ has all the leaves compact. Then the leaf space $N=M / \mathcal{D}$ inherits a structure of quaternion Kähler orbifold with positive scalar curvature.

Proof. Denote by $\Theta_{N}$ the projection of $\Theta$ on the leaf space. The vanishing of $\nabla \Theta_{N}$ can be obtained by computing $\nabla \Theta=\sum_{\alpha=1,2,3} \nabla\left(\Omega_{\alpha} \wedge \Omega_{\alpha}\right)$ on basic vector fields of the Riemannian submersion $M \rightarrow N$. One obtains:

$$
\nabla_{X} \Omega_{\alpha}(Y, Z)=g\left(Y,\left(\nabla_{X} I_{\alpha}\right) Z\right),
$$


and for basic $X, Y, Z$ the right-hand side vanishes (1.7.b). The parallelism of $\Theta_{N}$ then follows.

The result holds also for $\operatorname{dim} M=8$ (cf. Remark 3.9(i)). Note that, according to Proposition 2.3, the fibration $M \rightarrow N$ can never be trivial.

Remark 3.4. Any of the surfaces in Kato's list can actually occur as leaves of $\mathcal{D}$. To see this, just consider the standard hypercomplex Hopf manifold $S^{4 n-1} \times S^{1}=$ $H_{\mathbb{H}}=\left(\mathbb{H}^{n}-\{0\}\right) / \Gamma_{2}$ and the diagonal action of any $G$ in the list on the elements $\left(h_{0}, h_{1}, \ldots, h_{n-1}\right)$ of $\mathbb{H}^{n}-\{0\}$. In this way, $G$ acts on the fibers of $S^{4 n-1} \times S^{1} \rightarrow$ $\mathbb{H} P^{n-1}$, i.e. on the primary standard Hopf surface $S^{3} \times S^{1}$. Note that, except in case (i) for $m=1$, all fibers are nonprimary.

To better understand the above projection $p: M \rightarrow N$, we fix a complex structure $J \in H$ and consider the complex analytic foliation $\mathcal{V}_{J}$ spanned by $B$ and $J B$. From [Va2] and [Pe-Po-Sw] we then know that the leaf space $Z_{J}=M / \mathcal{V}_{J}$ is a KählerEinstein orbifold with positive scalar curvature. Let $\rho$ be the projection $M \rightarrow Z_{J}$.

Remark 3.5. Assume $\mathcal{D}$ to have all the leaves compact. Then for any $J \in H, Z_{J}$ is analytically equivalent with the twistor space $Z$ of $N$.

Proof. Fix $q \in Z_{J}$. Its counterimage on $M$ is a complex torus on a well-determined hypercomplex Hopf surface $S$, leaf of $\mathcal{D}$. If $r$ is the image of $S$ on $N$, then $q \mapsto r$ defines a Riemannian submersion $\pi: Z_{J} \rightarrow N$ and $p=\pi \circ \rho$. As the image of $S$ under $\rho$ is a sphere $S^{2}, \pi$ naturally realizes $Z_{J}$ as the total space of a $S^{2}$-bundle over $N$. The fixed complex structure $J$ on $M$ projects on $Z_{J}$ under $\rho$ but not on $N$ under $\pi$. However, once a $q$ in $Z_{J}$ is chosen, $J$ defines a compatible complex structure $K_{q} \in H_{r} \subset \operatorname{End}\left(T_{r} N\right)$ by: $K_{q} X=\pi_{*} J \widetilde{X}_{q}, \widetilde{X}_{q}$ being the horizontal lift at $q$ of $X \in T_{r} N$. This identifies the complex structure of $Z_{J}$ with that of the twistor space $Z$ of $N$.

We now consider the foliation $\mathcal{B}$ generated on $M$ by the Lee vector field $B$ and its orthogonal complement $\mathcal{B}^{\perp}$. The integrability of $\mathcal{B}^{\perp}$ is assured by $d \omega=0$.

Proposition 3.6. $\mathcal{B}^{\perp}$ is a totally geodesic Riemannian foliation. Its leaves have an induced structure of 3-Sasakian manifolds.

In fact, each generalized Hopf structure $\left(M, g, I_{\alpha}\right)$ induces a Sasakian structure on the leaves. The three Killing vector fields $I_{\alpha} B$ restricted to each leaf are related by the formulas

$$
g\left(I_{\lambda} B, I_{\mu} B\right)=\delta_{\lambda \mu}, \quad\left[I_{\lambda} B, I_{\mu} B\right]=\varepsilon_{\lambda \mu}^{\nu} I_{\nu} B .
$$

This is precisely the definition of a 3 -Sasakian structure. Then, using the results of [Va3]:

Corollary 3.7. If $\mathcal{B}$ has compact leaves, $M$ is a flat $S^{1}$-principal bundle over a 3 -Sasakian orbifold $P=M / \mathcal{B}$. The projection map is a Riemannian submersion.

On the other hand, by [Bo-Ga-Ma1], also 3-Sasakian manifolds fiber on quaternion Kähler orbifolds of positive scalar curvature with 3-dimensional homogeneous spherical space forms $S^{3} / \Gamma$ as fibers. Note that $\Gamma$ is then one of the groups: $\mathbb{Z}_{m}$, $m \geq 1, D_{4 m}, m \geq 2, T_{24}, O_{48}, I_{120}$ appearing in Kato's list. Also, observe that all the compact hypercomplex Hopf surfaces classified by Ma. Kato are diffeomorphic to $S^{3} / \Gamma$-bundles over $S^{1}$ ([Ka2], p. 95). By looking at the leaves of $\mathcal{D}$ and $\mathcal{B}$, we see then that the $S^{3} / \Gamma$ still appear as fibering any 3-Sasakian orbifold $P=M / \mathcal{B}$ 
which is the leaf space of a compact l.c.h.K. manifold $M$ having all the leaves of $\mathcal{B}$ compact. It follows:

Theorem 3.8. (i) Let $M$ be a compact locally conformal hyperkähler manifold and assume that the foliations $\mathcal{D}, \mathcal{V}, \mathcal{B}$ have compact leaves. Then we have the following commutative diagram of fibre bundles (and Riemannian submersions) in the orbifold category:

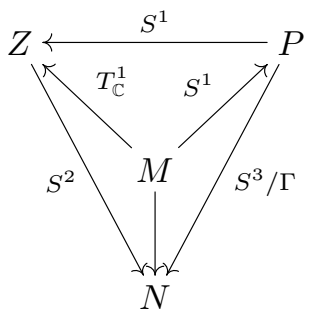

The fibres of $M \rightarrow N$ are Kato's integrable hypercomplex Hopf surfaces $\frac{S^{1} \times S^{3}}{G}$, not necessarily primary and not necessarily all homeomorphic.

(ii) Conversely, given a quaternion Kähler manifold $N$ of positive scalar curvature, there exists a commutative diagram as above with manifolds $M^{\prime}, Z^{\prime}, P^{\prime}$ respectively, locally conformal hyperkähler, Kähler-Einstein and 3-Sasakian and with fibers as described with $\Gamma=G=\mathbb{Z}_{2}$.

Proof. For the possibility of having nonprimary and nonequivalent fibers cf. Remark 3.4 and the example at the end of $\S 6$. As for statement (ii) consider the principal $S O(3)$-bundle $P^{\prime} \rightarrow N$ associated to $H \rightarrow N$. Then $P^{\prime}$ has an associated 3-Sasakian structure and any flat principal $S^{1}$-bundle $M^{\prime} \rightarrow P^{\prime}$ can be chosen to complete the diagram together with the twistor space of $N$. The obstruction to lifting $P^{\prime} \rightarrow N$ to a principal $S^{3}$-bundle is the Marchiafava-Romani class $\varepsilon \in H^{2}\left(N ; \mathbb{Z}_{2}\right)$ [Ma-Ro]. Note that all arrows appearing in the diagram are canonical, except $M \rightarrow Z$, which depends on the choice of a compatible complex structure on $M$.

Remark 3.9. (i) This diagram holds also if $\operatorname{dim}(M)=8$. In this case $N$ is still Einstein by the above discussion. The integrability of the complex structure on its twistor space implies it is also self-dual (cf. [Be]). Then just recall that a 4dimensional $N$ is usually defined to be quaternionic Kähler if it is Einstein and self-dual.

(ii) It is proved in [Bo-Ga-Ma1] that in every dimension $4 k-5, k \geq 3$, there are infinitely many distinct homotopy types of complete inhomogeneous 3-Sasakian manifolds. Thus, by simply making the product with $S^{1}$, we obtain infinitely many nonhomotopically equivalent examples of compact l.c.h.K. manifolds.

A first consequence of this diagram in Theorem 3.8 concerns cohomology. Here we restrict to manifolds, thus assuming all foliations regular in Palais' sense.

Note first that the property $\nabla \omega=0$ implies the vanishing $\chi(M)=0$ of the Euler characteristic. Then, applying twice the Gysin sequence in the upper triangle one finds the relations between the Betti numbers of $M$ and $Z$ [Va3]:

$$
\begin{aligned}
b_{i}(M) & =b_{i}(Z)+b_{i-1}(Z)-b_{i-2}(Z)-b_{i-3}(Z) \quad(0 \leq i \leq 2 n-1), \\
b_{2 n}(M) & =2\left[b_{2 n-1}(Z)-b_{2 n-3}(Z)\right] .
\end{aligned}
$$


On the other hand, since $N$ has positive scalar curvature, both $N$ and its twistor space $Z$ have zero odd Betti numbers [Be]. The Gysin sequence of the fibration $Z \rightarrow N$ then yields

$$
b_{2 p}(Z)=b_{2 p}(N)+b_{2 p-2}(N) .
$$

Together with (3.10) this implies

Theorem 3.11. Let $M$ be a compact locally conformal hyperkähler manifold with regular foliations $\mathcal{B}$ and $\mathcal{D}$. Then the following relations hold good:

$$
\begin{aligned}
& b_{2 p}(M)=b_{2 p+1}(M)=b_{2 p}(N)-b_{2 p-4}(N) \quad(0 \leq 2 p \leq 2 n-2), \\
& b_{2 n}(M)=0
\end{aligned}
$$

(Poincaré duality gives the corresponding equalities for $2 n+2 \leq 2 p \leq 4 n$ ). In particular $b_{1}(M)=1$. Moreover, if $n$ is even, $M$ cannot carry any quaternion Kähler metric.

Remark 3.12. (i) Under the hypotheses of Theorem 3.11 we obtain in particular $b_{2 p-4}(N) \leq b_{2 p}(N)$ for $0 \leq 2 p \leq 2 n-2$. Since any compact quaternion Kähler $N$ with positive scalar curvature can be realized as the quaternion Kähler base of a compact l.c.h.K. $M$ (Theorem 3.8(ii)), this implies, in the positive scalar curvature case, the Kraines-Bonan inequalities for Betti numbers of compact quaternion Kähler manifolds (cf. [Be]).

(ii) $b_{1}(M)=1$ is a much stronger restriction on the topology of compact l.c.h.K. manifolds in the larger class of compact generalized Hopf manifolds. For the latter the only restriction is $b_{1}$ odd and the induced Hopf bundles over compact Riemann surfaces of genus $g$ provide examples of generalized Hopf manifolds with $b_{1}=2 g+1$ for any $g$ [Va3].

Remark 3.13. The properties $b_{1}=1$ and $b_{2 n}=0$ have the following consequences:

(i) Let $\left(M, I_{1}, I_{2}, I_{3}\right)$ be a compact hypercomplex manifold that admits a locally and nonglobally conformally hyperkähler metric. Then none of the compatible complex structures $J=a_{1} I_{1}+a_{2} I_{2}+a_{3} I_{3}, a_{1}^{2}+a_{2}^{2}+a_{3}^{2}=1$, can support a Kähler metric. In particular, $\left(M, I_{1}, I_{2}, I_{3}\right)$ does not admit any hyperkähler metric.

(ii) Let $M$ be a $4 n$-dimensional $\mathcal{C}^{\infty}$ manifold that admits a locally and nonglobally conformal hyperkähler structure $\left(I_{1}, I_{2}, I_{3}, g\right)$. Then, for $n$ even, $M$ cannot admit any quaternion Kähler structure and, for $n$ odd, any quaternion Kähler structure of positive scalar curvature.

\section{The homogeneous CASE}

We call $M$ a locally conformal hyperkähler homogeneous manifold if there exists a Lie group which acts transitively and effectively on the left on $M$ by hypercomplex isometries.

Theorem 4.1. On a compact locally conformal hyperkähler homogeneous manifold the foliations $\mathcal{D}, \mathcal{V}$ and $\mathcal{B}$ are regular in Palais' sense and in diagram 3.8 all orbifolds $N, Z, P$ are homogeneous manifolds, compatibly with the respective structures.

Proof. Let $J \in H$ be a compatible complex structure on $M$. Then $(M, g, J)$ is a generalized Hopf homogeneous manifold and by [Va4, Theorem 3.2] we have the regularity of both the foliations $\mathcal{V}_{J}$ and $\mathcal{B}$. Therefore, $M$ projects on homogeneous manifolds $Z_{J}$ and $P$. In particular, the projections of $I_{\alpha} B$ on $P$ are regular Killing vector fields. Then Lemma 11.2 in [Ta] assures that the 3 -dimensional foliation 
spanned by the projections of $I_{1} B, I_{2} B, I_{3} B$ is regular. This, in turn, implies that $N$ is a homogeneous manifold; thus $\mathcal{D}$ is regular on $M$.

Note that Theorem 4.1 implies in particular that all the leaves of the foliations involved are compact (cf. [Pa]).

Starting with the Wolf classification of quaternion Kähler homogeneous manifolds, it is possible to classify 3-Sasakian homogeneous manifolds [Bo-Ga-Ma1]. This applies to our context and gives:

Proposition 4.2. The class of complex locally conformal hyperkähler homogeneous manifolds coincides with that of flat principal $S^{1}$-bundles over one of the following 3-Sasakian homogeneous manifolds: $S^{4 n-1}, \mathbb{R} P^{4 n-1}$, the flat manifolds $S U(m) / S(U(m-2) \times U(1)), m \geq 3, S O(k) /(S O(k-4) \times S p(1)), k \geq 7$, the exceptional spaces $G_{2} / S p(1), F_{4} / S p(3), E_{6} / S U(6), E_{7} / \operatorname{Spin}(12), E_{8} / E_{7}$.

The flat principal $S^{1}$-bundles over $P$ are characterized by having zero or torsion Chern class $c_{1} \in H^{2}(P ; \mathbb{Z})$ and are classified by it. The integral $H^{2}$ of the 3-Sasakian homogeneous manifolds can be computed by the following argument, suggested to the authors by Krzysztof Galicki. Look at the long homotopy exact sequence

$$
\cdots \rightarrow \pi_{2}(H) \rightarrow \pi_{2}(G) \rightarrow \pi_{2}(G / H) \rightarrow \pi(H) \rightarrow \pi(G) \rightarrow \cdots
$$

for the 3-Sasakian homogeneous manifolds $G / H$ listed in Proposition 4.2. Since $\pi_{2}(G)=0$ for any compact Lie group $G$, one obtains the following isomorphisms [Bo-Ga-Ma2]:

$$
H^{2}\left(\frac{S U(m)}{S(U(m-2) \times U(1)}\right) \cong \mathbb{Z}, \quad H^{2}\left(\mathbb{R} P^{4 n-1}\right) \cong \mathbb{Z}_{2}
$$

and $H^{2}(G / H)=0$ for all the other 3-Sasakian homogeneous manifolds. Combined with Proposition 4.2 this gives

Corollary 4.3. Let $M$ be a compact locally conformal hyperkähler homogeneous manifold. Then $M$ is one of the following:

(i) A product $(G / H) \times S^{1}$, where $G / H$ can be any of the 3 -Sasakian homogeneous manifolds $S^{4 n-1}, \mathbb{R} P^{4 n-1}, S U(m) / S(U(m-2) \times U(1)), m \geq 3, S O(k) /(S O(k-$ 4) $\times S p(1)), k \geq 7, G_{2} / S p(1), F_{4} / S p(3), E_{6} / S U(6), E_{7} / \operatorname{Spin}(12), E_{8} / E_{7}$.

(ii) The Möbius band, unique nontrivial principal $S^{1}$-bundle over $\mathbb{R} P^{4 n-1}$.

For example in dimension 8 one obtains $S^{7} \times S^{1}, \mathbb{R} P^{7} \times S^{1},\{S U(3) / S(U(1) \times$ $U(1))\} \times S^{1}$ and the Möbius band over $\mathbb{R} P^{7}$. The first exceptional example appears in dimension 12: the trivial bundle $\left\{G^{2} / S p(1)\right\} \times S^{1}$ whose 3-Sasakian base is diffeomorphic to the Stiefel manifold $V_{2}\left(\mathbb{R}^{7}\right)$ of the orthonormal 2-frames in $\mathbb{R}^{7}$.

\section{The LOCALLY CONFORMAL QUATERNION KÄHLER FIBRATION}

The structure Theorem 3.8 on compact locally conformal hyperkähler manifolds allows us to give a precise description of the more general case of compact locally conformal quaternion Kähler manifolds $M$. In fact, by referring to the fibrations of Theorem 2.1 we obtain:

Theorem 5.1. Let $M$ be a compact locally conformal quaternion Kähler manifold and assume that the foliation $\mathcal{D}$ has compact leaves. Then the base orbifold $N=M / \mathcal{D}$ is quaternion Kähler and the fibers are conformally flat 4-manifolds $(\mathbb{H}-\{0\}) / G$ where $G$ is a discrete subgroup of $G L(1, \mathbb{H}) \cdot S p(1)$ inducing an integrable quaternionic structure. 
The integrability of the quaternionic structure is here understood in the sense of the $G$-structures; i.e. an atlas of quaternionic coordinates is required to exist such that the jacobians of the changes of coordinates belong to $G L(1, \mathbb{H}) \cdot S p(1)$. Statement 5.1 then yields examples of such integrable structures. Note that, in accordance with a well-known result by Marchiafava-Kulkarni, the universal covering is an open subset of $\mathbb{H} P^{n}$ (cf. [Ma] or [Be], Theorem 14.64).

Proof. According to Remark 2.2, we choose on $M$ local hypercomplex structures $I_{1}, I_{2}, I_{3}$ integrable and parallel with respect to $D$. It follows that we may perform the same computations as in the l.c.h.K. case and locally project the bundle $H \subset \operatorname{End}(T M)$ on the Riemannian base $N$. Since $H$ is global on $M$, we obtain a quaternion Kähler orbifold $N$ of positive scalar curvature. Now let $F$ be a fiber of the above fibration. Due to the parallelism of $\omega$ it is a totally geodesic submanifold in $M$. We denote by $\omega_{0}$ (resp. $g_{0}$ ) the restriction of the Lee form (resp. l.c.q.K. metric $g$ ) to $F$ and by $B_{0}=\omega_{0}^{\natural}$. Around a point $x \in F$ we may consider a local orthonormal basis of tangent vector fields of the form $\left\{B_{0}, I_{1} B_{0}, I_{2} B_{0}, I_{3} B_{0}\right\}$. Using the parallelism of $\omega_{0}$ we easily compute the curvature tensor $R^{D}$ of the Weyl connection on this basis and obtain: $R^{D}\left(I_{\alpha} B_{0}, I_{\beta} B_{0}\right) I_{\gamma} B_{0}=0, R^{D}\left(I_{\alpha} B_{0}, I_{\beta} B_{0}\right) B_{0}=0$, $R^{D}\left(I_{\alpha} B_{0}, B_{0}\right) I_{\beta} B_{0}=0, R^{D}\left(I_{\alpha} B_{0}, B_{0}\right) B_{0}=0$. Thus, the curvature of the Weyl connection vanishes on $F$. This proves that $F$ is conformally flat. Furthermore, the well-known formula connecting the curvature tensors of two conformal metrics now gives the form of the curvature tensor $R_{0}$ (we again use the fact that $F$ is totally geodesic):

$$
\begin{aligned}
R_{0}(X, Y) Z= & \omega_{0}(X) \omega_{0}(Z) Y-\omega_{0}(Y) \omega_{0}(Z) X-\omega_{0}(X) g_{0}(Y, Z) B_{0} \\
& +\omega_{0}(Y) g_{0}(X, Z) B_{0}+g_{0}(Y, Z) X-g_{0}(X, Z) Y .
\end{aligned}
$$

It follows that the Ricci tensor $S_{0}=g_{0}-\omega_{0} \otimes \omega_{0}$ is $g_{0}$-parallel and that the sectional curvature is $\geq 0$ and strictly positive on an $\left\{I_{\alpha} B_{0}, I_{\beta} B_{0}\right\}$-plane. The universal Riemannian coverings of conformally flat Riemannian manifolds with parallel Ricci curvature have been classified by J. Lafontaine in [La]. By the above curvature discussion and the reducibility of $F$, due to $\nabla B_{0}=0$, we conclude that $\widetilde{F}$ is $\mathbb{R} \times S^{3}$, isometric with $\mathbb{R}^{4}-\{0\}$ with the conformally flat metric $g=(h \bar{h})^{-1} d h \otimes d \bar{h}$.

Riemannian manifolds with universal covering $\mathbb{R}^{n}-\{0\}$ have been considered in the contexts of local similarity manifolds [Va-Re] and, in dimension $n=4$, of Einstein-Weyl structures [Gau2]. In fact, it is proved in [Va-Re] that the curvature formula (5.2) implies that the allowed groups $G$ of covering transformations of $\mathbb{R}^{4}-\{0\}$ consists of conformal transformations

$$
\tilde{x}^{i}=\rho \sum_{j} a_{j}^{i} x^{j},
$$

where $\rho>0$ and $a_{j}^{i} \in S O(4)$. More precisely,

$$
G=\left\{h t_{0}^{k} ; h \in H, k \in \mathbb{Z}\right\},
$$

where $t_{0}$ is a conformal transformation with maximal module $0<\rho<1$ and $H$ is one of the finite subgroups of $U(2)$ classified by M. Kato [Ka1]. To conclude that $F$ has an induced integrable quaternionic structure one has only to recall that the conformal group $\mathrm{CO}^{+}$(4) of transformations (5.3) coincides with the 1-dimensional quaternionic group $G L(1, \mathbb{H}) \cdot S p(1)$. 
Accordingly, we call a real 4-dimensional Hopf manifold any quotient of $\mathbb{R}^{4}-\{0\}$ by one of the discrete groups $G \subset C O^{+}(4)$ described above. Therefore, the leaves $F$ of the foliation $\mathcal{D}$ on a compact l.c.q.K. manifold are such and, in particular, they may be complex Hopf surfaces (cf. Proposition 6.1) or hypercomplex Hopf surfaces as in the l.c.h.K. case.

\section{EXAMPLES: HYPERCOMPLEX AND QUATERNIONIC HOPF MANIFOLDS}

We now describe some natural examples of $4 n$-dimensional l.c.q.K. manifolds. We already noted that any real 4-dimensional Hopf manifold is an integrable quaternionic Hopf 4-manifold, i.e. a quotient of $\mathbb{H}-\{0\} \equiv \mathbb{R}^{4}-\{0\}$ by a discrete subgroup $G$ of $G L(1, \mathbb{H}) \cdot S p(1) \equiv C O^{+}(4)$. Moreover, since the metric $g=(h \bar{h})^{-1} d h \otimes d \bar{h}=$ $\left(\sum_{i} x_{i}^{2}\right)^{-1} \sum_{i}\left(d x_{i}\right)^{2}$ is invariant by the action of $G$, any real 4-dimensional Hopf manifold is a compact locally conformal quaternion Kähler manifold. More generally, consider the quaternion Hopf manifold $M=\left(\mathbb{H}^{n}-\{0\}\right) / G$, where $G$ is a discrete group of type (5.4) acting diagonally on the quaternionic coordinates $\left(h_{0}, \ldots, h_{n-1}\right)$. Endow $M$ with the metric $g=\left(\sum_{\alpha} h_{\alpha} \bar{h}_{\alpha}\right)^{-1} \sum_{\alpha} d h_{\alpha} \otimes d \bar{h}_{\alpha}$ and assume the foliation $\mathcal{D}$ to have compact leaves.

Proposition 6.1. The quaternion Hopf manifold $M=\left(\mathbb{H}^{n}-\{0\}\right) / G$ with the metric $g$ is a compact locally conformal quaternion Kähler manifold and the leaves $F$ of $\mathcal{D}$ are integrable quaternionic Hopf 4-manifolds. The leaf space $N$ is a quaternion Kähler orbifold quotient of $\mathbb{H} P^{n-1}$, whose set of singular points is generally the $\mathbb{R} P^{n-1} \subset \mathbb{H} P^{n-1}$. In particular:

(i) If $G$ is one of the groups listed in Kato's Theorem 3.2, then $M$ is a locally conformal hyperkähler manifold, the leaves $F$ of $\mathcal{D}$ are integrable hypercomplex Hopf surfaces, and $N$ is $\mathbb{H} P^{n-1}$.

(ii) If $G \subset G L(n, \mathbb{H}) \cdot U(1)$, e.g. $G=\Gamma_{c} \times \mathbb{Z}_{m}$ with $\Gamma_{c}$ acting on the left and $\mathbb{Z}_{m}$ on the right on the quaternionic coordinates, then the locally conformal quaternion Kähler manifold $M$ admits a compatible global complex structure and the leaves $F$ of $\mathcal{D}$ are complex Hopf surfaces. Here the set of singular points of the orbifold $N$ is the $\mathbb{C} P^{n-1} \subset \mathbb{H} P^{n-1}$.

Proof. In fact, in the general case, $G$ turns out to be a discrete subgroup of $G L(n, \mathbb{H}) \cdot S p(1)$, thus preserving the quaternionic structure of the universal covering. The structure of the leaves follows from Theorem 5.1. Since the two factors $G L(n, \mathbb{H})$ and $S p(1)$ act respectively on the left and on the right of the quaternionic coordinates of $\mathbb{H}^{n}-\{0\}$, the induced action of $G$ on the projective space $\mathbb{H} P^{n-1}$ identifies each point of it with finitely many other points, fixing those which can be represented in real coordinates. In the case (i), $G$ is a subgroup of $G L(n, \mathbb{H})$, thus preserving the hypercomplex structure of the universal covering and inducing the same structure on the leaves (cf. Proposition 3.1). The equality $N=\mathbb{H} P^{n-1}$ follows as in Proposition 3.3, where the group $\Gamma_{2}$ is replaced by $\Gamma_{c}$. The case (ii) is obtained by the inclusion $G L(n, \mathbb{H}) \cdot U(1) \subset G L(2 n, \mathbb{C})$, assuring the existence of a global integrable complex structure on $M$ and on the leaves of $\mathcal{D}$. These are real Hopf 4-manifolds with a complex structure, hence complex Hopf surfaces, as classified in [Ka1]. The induced action of $G$ in this case fixes all the points of complex coordinates.

We point out that quaternionic Kähler orbifolds that are finite quotients of $\mathbb{H} P^{n-1}$ may appear as leaf spaces $N=M / \mathcal{D}$ also of locally conformal hyperkähler 
$M=\left(\mathbb{H}^{n}-\{0\}\right) / \widetilde{G}$. Assume for example (cf. [Pe-Po-Sw], Remark 3.4) $n=2$ and $\widetilde{G}$ generated by $\left(h_{0}, h_{1}\right) \mapsto\left(2 e^{2 \pi i / 3} h_{0}, 2 e^{4 \pi i / 3} h_{1}\right)$. Then the leaf space $N=M / \mathcal{D}$ is a $\mathbb{Z}_{3}$-quotient of $\mathbb{H} P^{1}$. Note that the leaves of $\mathcal{D}$, standard Hopf surfaces $S^{3} \times S^{1}$ over the regular points of the orbifold, are nonprimary $\left(S^{1} \times S^{3}\right) / \mathbb{Z}_{3}$ only over the two singular points $[1: 0]$ and $[0: 1]$ of $N=\mathbb{H} P^{1} / \mathbb{Z}_{3}$.

\section{ACKNOWLEDGMENT}

The first author wishes to thank the Department of Mathematics of the University "La Sapienza" and his colleagues in Rome for their kind hospitality during the preparation of this paper.

\section{REFERENCES}

[Be] A. Besse, Einstein manifolds, Springer-Verlag, Berlin and New York, 1987. MR 88f: 53087

[Bo] Ch. P. Boyer, A note on hyperhermitian four-manifolds, Proc. Amer. Math. Soc. 102 (1988), 157-164. MR 89c:53049

[Bo-Ga-Ma1] Ch. P. Boyer, K. Galicki, and B. Mann, The geometry and topology of 3-Sasakian manifolds, J. Reine Angew. Math. 455 (1994), 183-220. MR 96e:53057

[Bo-Ga-Ma2] - Hypercomplex structures on Stiefel manifolds, Ann. of Global Anal. Geom. 14 (1996), 81-105. CMP 96:08

[Ch-Pi] B. Y. Chen and P. Piccinni, The canonical foliations of a locally conformal Kähler manifold, Ann. Mat. Pura Appl. 141 (1985), 289-305. MR 87e:53054

[Gau1] P. Gauduchon, La 1-forme de torsion d'une variété hermitienne compacte, Math. Ann. 267 (1984), 495-518. MR 87a:53101

[Gau2] Structures de Weyl-Einstein, espaces de twisteurs et variétés de type $S^{1} \times$ $S^{3}$, J. Reine Angew. Math. 469 (1995), 1-50. CMP 96:05

[Gr] A. Gray, A note on manifolds whose holonomy group is $S p(n) S p(1)$, Michigan Math. J. 16 (1969), 125-128. MR 39:6226

[Ka1] Ma. Kato, Topology of Hopf surfaces, J. Math. Soc. Japan 27 (1975), 222-238. MR 53:5949

[Ka2] _ Compact differentiable 4-folds with quaternionic structures, Math. Ann. 248 (1980), 79-96. MR 81h:53037

[Ku] Y. Y. Kuo, On almost contact 3-structures, Tôhoku Math. J. 22 (1970), 325-332. MR 43:3956

[La] J. Lafontaine, Remarques sur les variétés conformément plates, Math. Ann. 259 (1982), 313-319. MR 84a:53053

[Ma] S. Marchiafava, Sulle varietà a struttura quaternionale generalizzata, Rend. Mat. 3 (1970), 529-545. MR 43:2632

[Ma-Ro] S. Marchiafava and G. Romani, Sui fibrati con struttura quaternionale generalizzata, Ann. Mat. Pura Appl. 107 (1975), 131-157. MR 53:6558

[Mo] P. Molino, Riemannian foliations, Birkhäuser, Boston, 1988. MR 89b:53054

[Or] L. Ornea, Locally conformal Kähler manifolds. A survey, Quaderno n.12, Dip. Mat., Univ. di Roma "La Sapienza", 1994.

[Pa] R. S. Palais, A global formulation of the Lie theory of transformation groups Mem. Amer. Math. Soc. 22 (1957). MR 22:12162

[Pe-Po-Sw] H. Pedersen, Y. S. Poon, and A. Swann, The Einstein-Weyl equations in complex and quaternionic geometry, Diff. Geom. Appl. 3 (1993), 309-321. MR 94j:53058

[Pi] P. Piccinni, On the infinitesimal automorphisms of quaternionic structures, J. Math. Pures Appl. 72 (1993), 593-605. MR 94k:53048

[Sa] S. Salamon, Quaternionic Kähler manifolds, Invent. Math. 67 (1982), 143-171. MR 83k:53054

[Sat] I. Satake, The Gauss-Bonnet theorem for $V$-manifolds, J. Math. Soc. Japan 9 (1957), 464-476. MR 20:2022

[Ta] S. Tanno, Killing vectors on contact Riemannian manifolds and fiberings related to the Hopf fibration, Tôhoku Math. J. 23 (1971), 313-333. MR 44:4681 
[Ud] C. Udrişte, Structures presque coquaternioniennes, Bull. Math. Soc. Sci. Math. R.S. Roumanie 13 (1969), 487-507. MR 45:5908

[Va1] I. Vaisman, On locally conformal almost Kähler manifolds, Israel J. Math. 24 (1976), 338-351. MR 54:6048

[Va2] A geometric condition for an l.c.K. manifold to be Kähler, Geom. Dedicata 10 (1981), 129-134. MR 83b:53059

[Va3] $\quad$ Generalized Hopf manifolds, Geom. Dedicata 13 (1982), 231-255. MR 84g:53096

[Va4] _ A survey of generalized Hopf manifolds, in Differential Geometry on Homogeneous Spaces (Proc. Conf. Torino, Italy, 1983), Rend. Sem. Mat. Torini, Fasc. Spec., 205-221. MR 87g:53100

[Va-Re] I. Vaisman and C. Reischer, Local similarity manifolds, Ann. Mat. Pura Appl. 135 (1983), 279-291. MR 86b:53050

Faculty of Mathematics, University of Bucharest, 14, ACAdemiei Str., 70109 BuchaREST, ROMANia

E-mail address: lornea@imar.ro

Dipartimento di Matematica "G. Castelnuovo", Università di Roma "La Sapienza", P.Le A. Moro, 2, I-00185 Roma, Italy

E-mail address: piccinni@axrma.uniromas.it 